\title{
Entropic Graph Embedding via Multivariate Degree Distributions
}

\author{
Cheng Ye, Richard C. Wilson, and Edwin R. Hancock \\ Department of Computer Science, \\ University of York, \\ York, YO10 5GH, UK \\ \{cy666,richard.wilson, edwin.hancock\}@york.ac.uk
}

\begin{abstract}
Although there are many existing alternative methods for using structural characterizations of undirected graphs for embedding, clustering and classification problems, there is relatively little literature aimed at dealing with such problems for directed graphs. In this paper we present a novel method for characterizing graph structure that can be used to embed directed graphs into a feature space. The method commences from a characterization based on the distribution of the von Neumann entropy of a directed graph with the in and out-degree configurations associated with directed edges. We start from a recently developed expression for the von Neumann entropy of a directed graph, which depends on vertex in-degree and out-degree statistics, and thus obtain a multivariate edge-based distribution of entropy. We show how this distribution can be encoded as a multi-dimensional histogram, which captures the structure of a directed graph and reflects its complexity. By performing principal components analysis on a sample of histograms, we embed populations of directed graphs into a low dimensional space. Finally, we undertake experiments on both artificial and real-world data to demonstrate that our directed graph embedding method is effective in distinguishing different types of directed graphs.
\end{abstract}

Keywords: directed graph embedding, von Neumann entropy, entropy distribution.

\section{Introduction}

There has been a considerable body of work aimed at extracting features from undirected graphs which reflect their structure and complexity. With such features to hand, especially multi-dimensional ones, then problems such as graph embedding, clustering and classification can be addressed using standard machine learning and pattern recognition techniques. Unfortunately, there is very little work on the corresponding problems for directed graphs. This is unfortunate since many of the most common networks structures, e.g. the World Wide Web, exist in the form of directed graphs.

Motivated by the need to fill this gap in literature, in this paper we aim to develop a method based on information theory to extract multi-dimensional

P. Fränti et al. (Eds.): S+SSPR 2014, LNCS 8621, pp. 163-172, 2014.

(C) Springer-Verlag Berlin Heidelberg 2014 
features that can be used to characterize the structure of directed graphs, and hence render them amenable to embedding, clustering and classification. The starting point is a recent result where we have shown how to compute the von Neumann entropy for a directed graph using the configurations of in and outdegrees on directed edges.

\subsection{Related Literature}

Quantifying the intrinsic complexity of undirected graphs is a problem of fundamental practical importance in network analysis and pattern recognition. A good recent review of the state of the art can be found in the collection of papers edited by Dehmer and Mowshowitz [1]. Moreover, the entropy measures have also been shown to be an effective tool for representing the complexity in graph structure. Han et al. 2] have shown how to approximate the calculation of von Neumann entropy in terms of simple degree statistics rather than needing to compute the normalized Laplacian spectrum.

However, while the problem of computing the entropy of undirected graphs is well studied, the literature on directed graphs is rather limited. One recent exception is the work of Berwanger et al. [3], who have proposed a new parameter for the complexity of infinite directed graphs by measuring the extent to which cycles in graphs are intertwined.

We now turn our attention to embedding methods, which has become a topic of considerable interest for characterizing patterns and graphs in recent years. Broadly speaking, with different choices of graph structure characteristics, there are many existing alternative measures for embedding undirected graphs into feature vectors. An interesting method is provided by Ren et al. 4], who have used the polynomial coefficients determined by the Ihara zeta function to construct a feature vector, which shows good performance in graph clustering. Moreover, feature vectors can also be derived by embedding graphs into a feature space based on dissimilarity embedding [5. Unfortunately, there are relatively few corresponding methods developed for embedding directed graphs into a feature space. One exception is the work proposed by Chen et al. [6], who have suggested a directed graph embedding method by preserving the local information of vertices in a directed graph. Similarly, directed graph embedding can also be obtained by retaining the information of directionality of the graph [7].

\subsection{Contribution}

The motivation of this paper is to explore whether we can extract multidimensional structural features from directed graphs, and hence apply standard techniques from pattern recognition and machine learning to embed, cluster and classify data in the form of samples of directed graphs. One natural way of capturing the structure of a graph at the complexity level, is to use an entropic characterization. Hence we commence by computing the von Neumann entropy associated with each edge in a directed graph. An analysis, extending our own 
previously published work [8] shows that the entropy depends on the configuration of in and out-degrees of the two vertices defining a directed edge. This leads us to a four-dimensional characterization of directed graph structure, which depends on the distribution of entropy with the in and out-degrees of pairs of vertices connected by a directed edge. We represent this distribution by a fourdimensional histogram, which can be encoded as a long-vector for the purposes of analysis. To curb the size of the histogram, we show how to requantize the bin-contents using quantiles of the four cumulative degree distributions.

\section{Graph Embedding via Von Neumann Entropy Distribution}

In this section, we start from an approximation of the von Neumann entropy of a directed graph [8], and quantify the entropy associated with each directed edge. We show that this entropy is determined by the in and out-degrees of the start and end vertices connected by a directed edge. Based on this observation we explore the multivariate distribution of directed edge entropy with the different combinations of vertex in and out-degrees that define edges in a graph. In practice this distribution can be computed by constructing a multi-dimensional histogram whose bins are indexed by the in and out-degrees of the connected vertices and whose contents accumulates the entropy contributions over the directed edges in the graph. The contents of the histogram can be represented by a multi-dimensional array whose contents can be encoded as a long-vector, which serves as a feature vector for the graph.

One of the problems that potentially limits this approach is that the vertex degree is unbounded. Hence, the size of histogram can become large. Moreover, it can become populated by a large number of empty bins. This renders the analysis of the feature vector unstable. In order to keep the vector length constant and reduce the number of empty bins, we requantize the degree bins of the histogram using quantiles of the cumulative distribution function (CDF). Specifically, we determine the $m$-quantiles, which divides the ordered vertex degree data into $m$ essentially equal-sized parts. This allows us to relabel each vertex with two quantile labels $(1,2, \ldots, m)$, one for in-degree and the second for out-degree. As a result, the length of our proposed feature vector is not affected by the variance of the degree distribution.

\subsection{Edge-Based Local Entropic Measure}

Suppose $G(V, E)$ is a directed graph with vertex set $V$ and edge set $E \subseteq V \times V$, then the adjacency matrix $A$ is defined as follows

$$
A_{u v}= \begin{cases}1 & \text { if }(u, v) \in E \\ 0 & \text { otherwise }\end{cases}
$$

The in-degree and out-degree of vertex $u$ are

$$
d_{u}^{\text {in }}=\sum_{v \in V} A_{v u} \quad d_{u}^{\text {out }}=\sum_{v \in V} A_{u v}
$$


Recently, commencing from Passerini and Severini's work 9], Ye et al. 8] have extended the calculation of von Neumann entropy from undirected graphs to directed graphs, using Chung's definition of the normalized Laplacian of a directed graph [10, with the result that

$$
H_{V N}^{D}=\frac{1}{2|V|}\left\{\sum_{(u, v) \in E} \frac{d_{u}^{\text {in }}}{d_{v}^{\text {in }} d_{u}^{\text {out }}}+\sum_{(u, v) \in E_{b}} \frac{1}{d_{u}^{\text {out }} d_{v}^{\text {out }}}\right\}
$$

where $E_{b}=\{(u, v) \mid(u, v) \in E$ and $(v, u) \in E\}$ is the set of bidirectional edges.

In particular, if the cardinality of $E_{b}$ is very small $\left(\left|E_{b}\right| \ll|E|\right)$, i.e. a graph is strongly directed (SD), this expression can be simplified one step further by ignoring the summation over $E_{b}$ in Eq.(3),

$$
H_{V N}^{S D}=\frac{1}{2|V|} \sum_{(u, v) \in E}\left\{\frac{d_{u}^{i n}}{d_{v}^{i n} d_{u}^{o u t^{2}}}\right\}
$$

These approximations sum the entropy contribution from each directed edge, and these are based on the in and out-degree statistics of the directed edge. In other words we can compute a normalized local entropy measure for each directed edge. Specifically, for an edge $(u, v) \in E$, we compute

$$
I_{u v}=\frac{d_{u}^{i n}}{2|E||V| d_{v}^{\text {in }} d_{u}^{\text {out }}{ }^{2}}
$$

as the entropy contribution. If this edge is bidirectional, i.e. $(u, v) \in E_{b}$, then we add an addition entropy contribution

$$
I_{u v}^{\prime}=\frac{1}{2\left|E_{b}\right||V| d_{u}^{\text {out }} d_{v}^{\text {out }}}
$$

This local measure represents the entropy associated with each directed edge since for arbitrary directed graphs, we have $\sum_{(u, v) \in E} I_{u v}+\sum_{(u, v) \in E_{b}} I_{u v}^{\prime}=H_{V N}^{D}$ and for strongly directed graphs, we also have $\sum_{(u, v) \in E} I_{u v}=H_{V N}^{S D}$. Moreover, this measure avoids the bias caused by graph size, which means that it is the edge entropy contribution determined by the in and out-degree statistics, and neither the vertex number or edge number of the graph that distinguishes a directed edge.

\subsection{Feature Vector Extracted from Entropy Distribution}

Our directed graph characterization is based on the statistical information converged by the distribution of directed edge entropy with the in and out-degrees of the start and end vertices. We represent this distribution of entropy using a four-dimensional histogram over the in and out-degrees of the two vertices.

As noted above, one potential problem is that the bin-contents can become sparse in a high dimensional histogram. To overcome this problem we turn to the cumulative distribution function. Suppose a directed graph $G(V, E)$ has $|V|$ 
vertices which have been sorted according to in-degree (or out-degree) in the sequence $d_{1}^{i n} \leq d_{2}^{i n} \leq \cdots \leq d_{|V|}^{i n}$. Let $P\left(X=d_{i}^{i n}\right)$ be the in-degree probability distribution of the graph. The corresponding cumulative distribution function for the in-degree is given by

$$
F_{X}\left(d_{i}^{i n}\right)=P\left(X \leq d_{i}^{i n}\right)
$$

where $i=1,2, \ldots,|V|$. This function describes the probability that a given indegree $X$ takes on a value less than or equal to $d_{i}^{i n}$.

Quantiles are intervals of equal size over the cumulative distribution function. They divide the ordered data $d_{1}^{i n}, d_{2}^{i n}, \cdots, d_{|V|}^{i n}$ into a number of equal-sized data subsets. Since vertex degree is always a non-negative integer, the quantiles can thus be viewed as new quantization of the degree based on its statistical distribution. We define our degree quantiles over the cumulative distribution of degree for the entire sample of graphs under study, and produce requantized versions of the individual entropy histograms for each individual graph. Suppose the number of quantiles in each dimension of the degree distribution is fixed to be $m$. Then, for example, the $m$-quantiles of the in-degree distribution can be obtained as follows

$$
Q_{j}=\underset{d_{i}^{i n}}{\operatorname{argmin}}\left\{F_{Q_{j}}\left(d_{i}^{i n}\right)-\frac{j}{m}\right\}
$$

where $i=1,2, \ldots,|V|$ and $j=1,2, \ldots, m$. It is clear that these degree quantiles satisfy $Q_{1} \leq Q_{2} \leq \cdots \leq Q_{m}$ and in fact, $Q_{m}=d_{|V|}^{i n}$.

With the sample degree quantiles to hand, we assign each vertex degree quantile labels. We first examine the original in-degree $d_{u}^{i n}$ of a vertex $u$, if $d_{u}^{\text {in }}$ satisfies the condition that $Q_{k-1}<d_{u}^{i n} \leq Q_{k}$, then its in-degree quantile is $q_{u}^{i n}=k$. The corresponding out-degree quantile labels can also be obtained in the same manner. Since all the vertices in the graph have in-degree and out-degree quantile labels ranging from 1 to $m$, we can then simply construct the directed edge entropy histogram whose size in each dimension is fixed to $m$. The histogram is stored as a four-dimensional array.

To do this, we first construct a $m \times m \times m \times m$ array $M$ whose elements represent the histogram bin-contents, and whose indices represent the degree quantile labels of the vertices. For instance, the element $M(1,2,3,4)$ accumulates the entropy contribution for all the directed edges starting from vertices with out-degree quantile label 1 and in-degree quantile label 2, pointing to vertices with out-degree quantile label 3 and in-degree quantile label 4 . We then compute the bin-contents by summing the directed edge entropy contributions over the sample graph. The histogram bins contain all directed edges having the same quantile label combinations. We store the accumulated sum in the corresponding element of array $M$. The elementwise accumulation is formally given as

$$
M_{i j k l}=\sum_{\substack{q_{u}^{\text {out }}=i, q_{u}^{\text {in }}=j \\ q_{v}^{\text {out }}=k, q_{v}^{\text {in }}=l \\(u, v) \in E}}\left\{\frac{d_{u}^{\text {in }}}{2|E||V| d_{v}^{\text {in }} d_{u}^{\text {out }}{ }^{2}}\right\}
$$


If the graph contains bidirectional edges, we additionally accumulate the following quantity

$$
M_{i j k l}^{\prime}=\sum_{\begin{array}{c}
q_{u}^{\text {out }}=i, q_{u}^{\text {in }}=j \\
q_{v}^{\text {out }}=k, q_{v}^{\text {in }}=l \\
(u, v) \in E_{b}
\end{array}}\left\{\frac{1}{2\left|E_{b}\right||V| d_{u}^{\text {out }} d_{v}^{\text {out }}}\right\}
$$

where $i, j, k, l=1,2, \ldots, m$. To extract a feature vector from $M$, we can simply list all the elements in the array, with the result that

$$
v=\left(M_{1111}, M_{1112}, \cdots, M_{111 m}, M_{1121}, M_{1122}, \cdots, M_{m m m m}\right)^{T}
$$

Clearly, this feature vector has length $m^{4}$.

It is worth pausing to consider the case of strongly directed graphs. For such graphs, from Eq.(4) it is clear that directed edge entropy does not depend on $d_{v}^{\text {out }}$. As a result the dimensionality of the corresponding histogram can be reduced from four to three by ignoring the third index $k$ in $M_{i j k l}$ (Eq.(8)). This leads to a new feature vector with length $\mathrm{m}^{3}$. In the following discussion, to distinguish between these two kinds of feature vectors, we name the former full-form (FF) while the latter strongly-directed (SD).

When accumulated in this way we effectively count directed edges with the same configurations of degree quantile labels, and weight them according to their entropy. If the different quantile labels were independent, we would expect a uniform histogram. However, structure in the individual sample graphs due to preferred combinations of vertex in-degree and out-degree will give rise to a non-uniform distribution. To some extent, the quantization of the distribution of entropy with degree according to quantile labels, may dilute this structure due to merging adjacent degree bins. However, the directed edge entropy contribution is based on the original vertex in and out-degree statistics, and the $m$-quantiles play a role in diminishing the bias caused by different populations of directed graphs. Therefore our proposed representation can still be effective in capturing statistical information concerning the local structural properties in the graph. By embedding graphs into a space spanned by feature vectors, it provides a theoretically principled and efficient tool for graph characterization tasks, which captures the graph characteristics at both the statistical and structural levels.

\section{Experiments and Evaluations}

In this section, we aim to evaluate the experimental performance of our suggested directed graph characterization. Specifically, we first explore the graph clustering performance of our method on a set of random graphs generated from three classical random graph models. Then we apply our method to some real-world data, including the COIL object recognition data and protein database, and report the graph classification results. 


\subsection{Datasets}

We commence by giving a brief overview of the datasets used for experiments in this paper. We use three different datasets, the first one is synthetically generated artificial networks, while the other two are extracted from real-world systems.

Artificial Data: Contains a large number of directed graphs which are randomly generated according to a) the classical Erdös-Rényi model, b) the "small-world" model, and c) the "scale-free" model. The different graphs in the database are created using a variety of model parameters, e.g. the graph size and the vertex connection probability in the Erdős-Rényi model, the edge rewiring probability in the "small-world" model and the number of added connections at each time step in the "scale-free" model.

COIL Data: Contains object recognition data collected by Nene et al. [1], in which each 3D object consists of 72 images collected from equally spaced changes in viewing direction over 360 degrees. For each image, we establish a 3 -nearest neighbour graph on the extracted feature points, i.e. each feature point have three directed edges going to its nearest neighbour points, thus the graph is directed and the out-degree of all vertices is 3 . There are two subsets in this database, one contains the directed graphs extracted from 4 different 3D objects while the other contains graphs from 8 objects.

Protein Data: Is extracted from the protein database previously used by Riesen and Bunke 12. It consists of over 200 graphs, representing proteins labelled with their corresponding enzyme class labels from the BRENDA enzyme database. The database consists of six classes (labelled EC 1, ., EC 6), which represent proteins out of the six enzyme commission top level hierarchy (EC classes). The proteins are converted into graphs by first replacing the secondary structure elements of a protein with vertices, and then constructing a 3-nearest neighbour graph for the secondary structure elements. The graphs are thus directed.

\subsection{Graph Clustering Performance}

To investigate the clustering performance of our proposed directed graph characterization, we perform principle component analysis (PCA) on both FF feature vectors and SD feature vectors extracted from the randomly generated graphs in the Artificial Data. These feature vectors are long-vectors formed by concatenating the elements of the four and three-dimensional histograms respectively. Here we select different parameter settings to generate 500 normal directed graphs and 500 additional strongly directed graphs for each of the three random graph models, with graph size ranged between 100 and 150. Moreover, in all the experiments in this section, we choose the number of quantiles $m=3$, giving all the FF feature vectors with a constant length $m^{4}=81$, while for SD feature vectors, the length is $m^{3}=27$.

Figures 1(a), (c) and (d) each show that by embedding different random graphs into a feature space spanned by the first three principal components constructed from the feature vectors, the three classes of random graphs display 
some clear separation between each other. However in Fig.1(b), which is the plot of SD feature vectors extracted from normal directed graphs, the "small-world" graphs and "scale-free" graphs show some overlap. This suggests the FF feature vectors are efficient in distinguishing any normal directed graphs while the SD feature vectors are effective only for strongly directed graphs, which is an expected result. Therefore in the following experiments we use the FF feature vectors in our analysis.

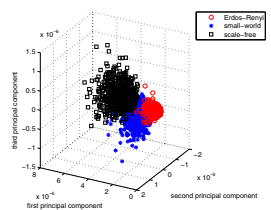

(a)

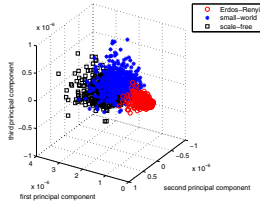

(b)

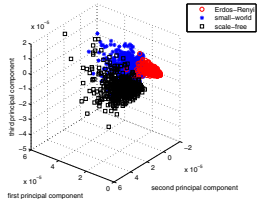

(c)

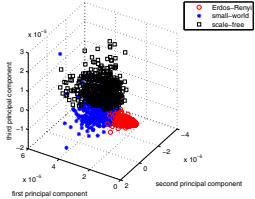

(d)

Fig. 1. Clustering performance for random graphs using PCA: a) FF feature vectors extracted from normal directed graphs; b) SD feature vectors extracted from normal directed graphs; c) FF feature vectors extracted from SD graphs; d) SD feature vectors extracted from SD graphs. Red: Erdős-Rényi graphs; blue: "small-world" graphs; black: "scale-free" graphs.

\subsection{Graph Classification Results}

To take this analysis one step further, we evaluate the classification performance of our method on the graphs in COIL DATA and Protein Data, using standard vector-based clustering and classification algorithms. In the following evaluation, we perform the 10-fold cross-validation using two classifiers, namely support vector machine (SVM) classifier associated with the sequential minimal optimization (SMO) 13 and the Pearson VII universal kernel (Puk), and k-nearest neighbour $(\mathrm{kNN})$ classifier. All the SMO-SVM and kNN parameters are optimized for each method on a Weka platform, and all experiments are performed on an Intel(R) Core(TM) i7-3770 CPU @ $3.40 \mathrm{GHz}$ processor, with 8 GB memory.

In Fig. 2 we report the average classification rates of 10 runs for both SVM and kNN classifiers as a function of quantile number $m$ on three different datasets, including the 4-object data and 8-object data in COIL Data and Protein Data. Figure 3 gives the relationship between the average runtime and the quantile number of the experiments on these datasets.

From Fig.3 we find that the experimental runtime for all three classification problems grows as the quantile number increases, which is as expected since greater quantile number leads to greater size of the feature vector, resulting in the greater computational complexity. Moreover, it is clear that our directed graph characterization is computationally tractable as the runtime does not increase rapidly even when the size of the feature vector becomes particularly large. 


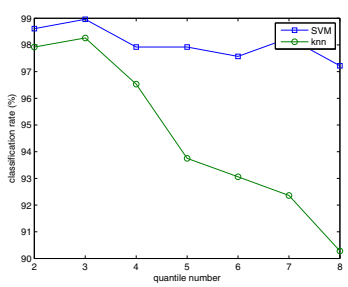

(a)

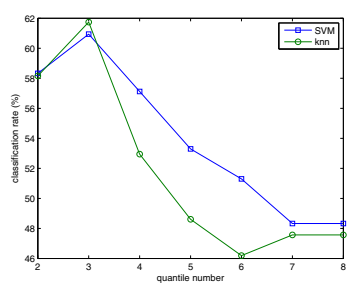

(b)

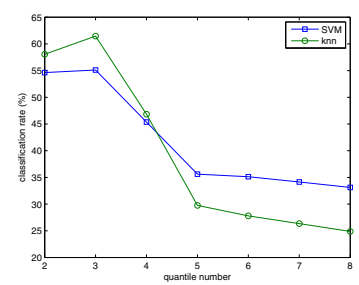

(c)

Fig. 2. Average classification rates for both SVM and kNN classifiers with different quantile numbers on datasets: a) 4-object data; b) 8-object data and c) Protein Data. Square: SVM classifier; circle: kNN classifier.

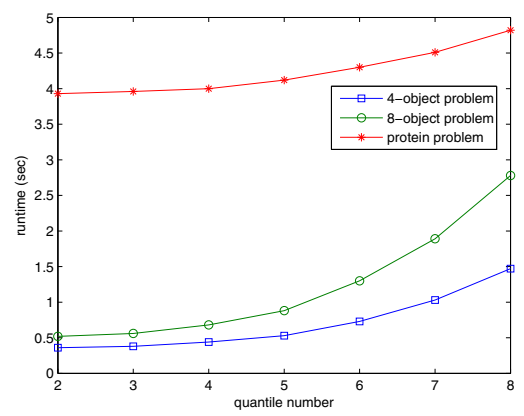

Fig. 3. Average experimental runtime with various quantile numbers for different classification problems. Square: 4-object problem; circle: 8-object problem; star: protein problem.

Turning attention to the classification results reported in Fig.2(a), (b) and (c), we find the performance is particularly good on 4-object data, with a classification accuracy over 98\%, and on 8-object data and 6-class protein database, the accuracy is still acceptable (50\% to $60 \%)$. Moreover, as the increase of the quantile number, the classification rates for both classifiers on all three datasets witness a slight growth, reaching a peak when the quantile number reaches 3 , then they drop significantly. This is because in the graphs of these datasets, all vertices have the same out-degree 3 , therefore when $m=3$ the corresponding feature vectors can precisely preserve the information of the vertex in and out-degree statistics, which guarantees that $m=3$ gives the best classification performance and any greater quantile number will lead to a decrease of classification accuracy. Furthermore, with this choice of quantile number, the experimental runtime is relatively low, which suggests that our method can achieve a sufficient accuracy without causing expensive computation. Overall, based on these observations we claim that that our directed graph characterization can be both accurate and computationally efficient in clustering and classifying directed graphs when the appropriate parameters are selected. 


\section{Conclusion}

In this paper we have suggested a novel and effective method for directed graph characterization based on the multivariate distribution of local von Neumann entropy contribution with vertex in-degree and out-degree. This provides a complexity level characterization of graph structure based on the statistical information residing edge degree distribution. By representing graphs using feature vectors that encode the entropy distribution, both clustering and classification can be addressed using standard pattern recognition and machine learning techniques. We have undertaken experiments to demonstrate that our method is both accurate and computationally efficient in dealing with both artificial and real-world data. In the future, we intend to explore kernels defined over the inner products of our entropy distribution feature vectors.

\section{References}

1. Dehmer, M., Mowshowitz, A., Emmert-Streib, F.: Advances in Network Complexity. Wiley-Blackwell (2013)

2. Han, L., Escolano, F., Hancock, E., Wilson, R.: Graph characterizations from von neumann entropy. Pattern Recognition Letters 33, 1958-1967 (2012)

3. Berwanger, D., Gradel, E., Kaiser, L., Rabinovich, R.: Entanglement and the complexity of directed graphs. Theoretical Computer Science 463, 2-25 (2012)

4. Ren, P., Wilson, R., Hancock, E.: Graph characterization via ihara coefficients. IEEE Transactions on Neural Networks 22, 233-245 (2011)

5. Bunke, H., Riesen, K.: Improving vector space embedding of graphs through feature selection algorithms. Pattern Recognition 44, 1928-1940 (2010)

6. Chen, M., Yang, Q., Tang, X.: Directed graph embedding. In: IJCAI, pp. 2707-2712 (2007)

7. Perrault-Joncas, D., Meliă, M.: Directed graph embedding: an algorithm based on continuous limits of laplacian-type operators. Advances in Neural Information Processing Systems 24, 990-998 (2011)

8. Ye, C., Wilson, R.C., Comin, C.H., da F. Costa, L., Hancock, E.R.: Entropy and heterogeneity measures for directed graphs. In: Hancock, E., Pelillo, M. (eds.) SIMBAD 2013. LNCS, vol. 7953, pp. 219-234. Springer, Heidelberg (2013)

9. Passerini, F., Severini, S.: The von neumann entropy of networks. Inthernational Journal of Agent Technologies and Systems, 58-67 (2008)

10. Chung, F.: Laplacians and the cheeger inequailty for directed graphs. Annals of Combinatorics 9, 1-19 (2005)

11. Nene, A., Nayar, S., Murase, H.: Columbia object image library (coil-20). Technical Report (CUCS-005-96) (February 1996)

12. Riesen, K., Bunke, H.: IAM graph database repository for graph based pattern recognition and machine learning. In: da Vitoria Lobo, N., Kasparis, T., Roli, F., Kwok, J.T., Georgiopoulos, M., Anagnostopoulos, G.C., Loog, M. (eds.) SSPR\&SPR 2008. LNCS, vol. 5342, pp. 287-297. Springer, Heidelberg (2008)

13. Platt, J.: Sequential Minimal Optimization: A Fast Algorithm for Training Support Vector Machines. MIT Press (1999) 